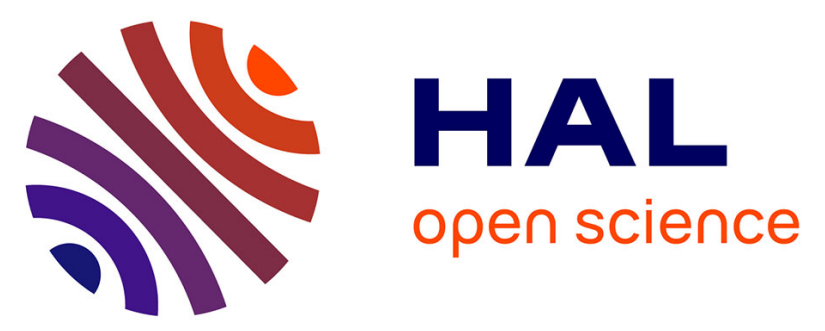

\title{
A Case Study on the Analysis of Behavior Patterns and Pattern Changes in Smart Environments
}

\author{
Paula Lago, Claudia Jiménez-Guarín, Claudia Roncancio
}

\section{To cite this version:}

Paula Lago, Claudia Jiménez-Guarín, Claudia Roncancio. A Case Study on the Analysis of Behavior Patterns and Pattern Changes in Smart Environments. 6th International Work-Conference on Ambient Assisted Living and Daily Activities (IWAAL 2014), Dec 2014, Belfast, Ireland. pp.296-303. hal-01659778

\section{HAL Id: hal-01659778 https://hal.science/hal-01659778}

Submitted on 23 Jan 2018

HAL is a multi-disciplinary open access archive for the deposit and dissemination of scientific research documents, whether they are published or not. The documents may come from teaching and research institutions in France or abroad, or from public or private research centers.
L'archive ouverte pluridisciplinaire HAL, est destinée au dépôt et à la diffusion de documents scientifiques de niveau recherche, publiés ou non, émanant des établissements d'enseignement et de recherche français ou étrangers, des laboratoires publics ou privés. 


\title{
A Case Study on the Analysis of Behavior Patterns and Pattern Changes in Smart Environments
}

\author{
Paula Lago ${ }^{1}$, Claudia Jiménez-Guarín ${ }^{1}$, and Claudia Roncancio ${ }^{2}$ \\ ${ }^{1}$ Systems and Computing Engineering Department, School of Engineering, \\ Universidad de los Andes, Colombia \\ \{pa. lago52, cjimenez\}@uniandes. edu.co \\ ${ }^{2}$ Univ. Grenoble Alpes, LIG, France \\ claudia.roncancio@imag. fr
}

\begin{abstract}
Societies need to devise mechanisms of caring for the well aging of the increasing number of seniors, as it is very important for elderly people to maintain their independence. Smart environments are being devised as a form of care in what has been called ambient assisted living. A smart environment should be able to respond in case of emergency or risk and inform any abnormal behavior. Still, not much research is done to understand behavior patterns, temporal changes and other particularities that can affect the effectiveness of smart environments in ambient assisted living. We explored the behavior of two adults in a smart environment in order to reveal temporal, spatial and sequential relations among the activities as well as the changes that these relations undergo overtime and across individuals. This paper presents an analysis of three human behavior patterns: temporal, location and frequency. These patterns are mined on two experimental subjects using the dataset provided by the CASAS project.

Our analysis evidences how temporal, spatial and sequential patterns differ from person to person, day to day and after some time. Learning personalized behaviors and identifying and adapting to changes is a crucial aspect for smart environments since one-fit-all solutions are not suitable.
\end{abstract}

Keywords: behavior analysis, patterns, activities of daily living, elder care.

\section{Introduction}

Socio-economic development around the world has led to improvements in lifeexpectancy which, together with lower fertility rates, are changing the demographic structure of countries. Population is now older than ever before and people over 60 years represented in $20108 \%$ of the total world population [1]. These numbers are expected to grow even more in the years to come, posing challenges to societies in order to help elders maintain their autonomy and independent living for as long as possible. Assisted Living based on ambient intelligence (Ambient Assisted Living, AAL) is proposed as a way to enable independent living while still giving elders the proper support in cases of disease, disability or emergency [2].

There are many commercial [3, 4] and academic [5, 6] projects working towards AAL that will enable elders to live independently and safe at their own home. These

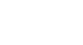


systems monitor activities of daily living in order to tell routine compliance, or detect some early signs of disease and/or risk situations that should be watched over.

It is agreed that AAL solutions could benefit from a better understanding of activity dynamics, behavior patterns and routines [7] and that changes in behavior trends could reveal a decline in health. In the field of gerontology, some studies have been made to understand activity patterns of seniors. Pruchno and Rose [8] study single day activities for a large population. This study shows variations as a function of personal characteristics, social context and environmental context. A similar study [9] concludes that obligatory activities are done in the morning while leisure activities are more common during the evenings. However, these studies don't consider long-term patterns of single individuals or frequent activity sequences or days' correlation or behavior evolution.

In this work, we present a case study for analyzing patterns of behavior of two subjects in a real-life setting with respect to some contextual variables: time, day of the week and location. We analyze the frequency of each activity and frequent sets of activities at the light of changes in each of these variables. This allows us to visualize and reinforce three hypotheses that result from intuition:

1. People have different routines and behavior patterns. Consequently we require personalized classifiers for activity recognition. For each person we can learn what features better describe an activity,

2. People behave differently on different days of the week. This means that day to day differences may not be abnormal and that classifiers can base their knowledge on a weekly correlation,

3. Routines change overtime, thus, one-fit-all solutions are not suitable for this problem.

This paper is organized as follows: Section 2 provides considerations for the analysis in our work. It also presents the datasets used for this analysis. Section 3 presents the analysis performed together with the results and findings. Section 4 summarizes the findings, presents some conclusions and future work to be done.

\section{Behavior Analysis in Smart Environments}

In this section we present the considerations made prior to the behavior analysis and the data used for the case study.

\subsection{Considerations for Behavior Analysis to Discover and Understand Routines}

A first step when creating a smart environment for ambient assisted living is to understand the activities and routines of its inhabitants.

In our approach, an activity is the fundamental semantically described unit, and it is why we used activities and not sensor data to analyze patterns. An activity is defined by its context: start time, duration and location, and by a goal, which defines its label. When activities occur in similar contexts, i.e. at the same times, on the same locations or followed by the same activities, we can infer patterns. Recurrent patterns lead to routines, allowing us to infer usual or unusual situations. 
Inferred patterns can change over time. Health conditions, new doings, new friends, new interests can happen on any time, making the learned routines and patterns obsolete. The models describing the individual daily life must be adaptable to the evolving situations, considering new patterns as normal when they arise.

We want to analyze these patterns and their changes to construct a semantically enriched context that can be used to create recommendations, alerts or notifications, both to the individual and to her care-givers, respecting the individual's privacy.

\subsection{Data Description}

For this study we used the Aruba ( $1^{\text {st }}$ subject in the paper) and Cairo ( $2^{\text {nd }}$ subject in the paper) CASAS Dataset which are made public by the CASAS project [5] through their repository (http://ailab.wsu.edu/casas/datasets.html). Both datasets consist of sensor data that was collected in the home of a volunteer adult with annotated activities. We used these datasets because they have data for long periods of time, are collected in real-life settings, and share similar activities that enable comparison. We used only two persons since it is hard to find other datasets with the same features.

The first dataset contains data from 2010-11-04 to 2011-06-11 (193 days) and the second dataset contains data from 2009-06-10 to 2009-08-05 (56 days). The annotated activities are ${ }^{1}$ : meal preparation, sleeping, relax, wash dishes, work, bed to toilet, enter and leave home, housekeeping and resperate ${ }^{2}$. The last activity is not considered in this work. Also, enter and leave home activities are re-labeled as not at home.

Each record in the dataset contains a timestamp, a sensor id, a sensor reading and a label, if an activity is starting or ending (see Fig. 1 (a)). Since we are concerned with studying spatial, temporal and sequence patterns of the activities and not with the sensor activations, we first transformed the dataset in order to have records that contain the start and end time of the activity, the location and the label (see Fig. 2(b)).

2010-11-04 00:03:50.209589 M003 ON Sleeping begin
2010-11-04 00:03:57.399391 M003 OFF
$2010-11-04$ 00:15:08.984841 T002 21.5
$2010-11-04$ 05:40:40.482626 M003 OFF
$2010-11-04$ 05:40:40.844463 M003 ON
$2010-11-04$ 05:40:43.642664 M003 OFF Sleeping end

(a)

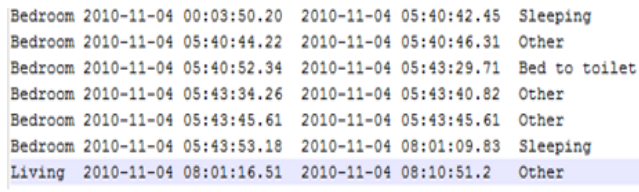

(b)

Fig. 1. (a) Original dataset and, (b). transformation done for this paper We used data that defines the location, start time and end time for each activity.

The location of each activity was defined as the room with the more sensor events during the time of the activity. The distribution of the sensors is available with each dataset.

\footnotetext{
1 Meal preparation, relax and wash dishes are not annotated for the second subject. Also, housekeeping refers to only laundry in the second subject.

2 Resperate is a device used to lower blood pressure.
} 


\section{Analysis of Behavioral Patterns Based on Time and Location}

This section presents the method and results for the analysis of three kinds of patterns that emerge from the data used: temporal patterns by days and hours, location patterns and activity sets, that is, activities that often occur one after the other.

\subsection{Temporal Patterns}

To study how activity probabilities correlate with temporal characteristics, we performed in a first step a qualitative analysis of the data the usual start times of each monitored activity. Figure 2 shows, for subject 2, the hour at which each of the activities started activity for different days of the week. Figure 3 shows, for subject 1 , the same information for different months of the years.

On one hand, the analysis of the data of subject 2 showed that while some activities exhibit similar start times for every day, others show clear differences depending on the day of the week they are done. For instance (Fig. 2), the first meal of the day is taken earlier on Wednesdays than on Sundays. Also, laundry is done earlier on Sundays. Clearly, the day of the week can change the start time of some activities.

On the other hand, the analysis of the data of subject 1 showed that routines, in the sense of start times for activities, change during the course of the year. When studied in two different periods of the year, housekeeping and washing dishes are almost nonexistent for the second period (Fig. 3). Also, during the second period eating is only done in the mornings. These changes could be alerted to a care-giver or, if they correspond to a new routine, they should be learned by the system

We also compared quantitatively the differences in the start times for each activity depending on the day of the week with two-proportion z- tests. For the test we calculated the probability of doing each activity at each hour of the day for each day.
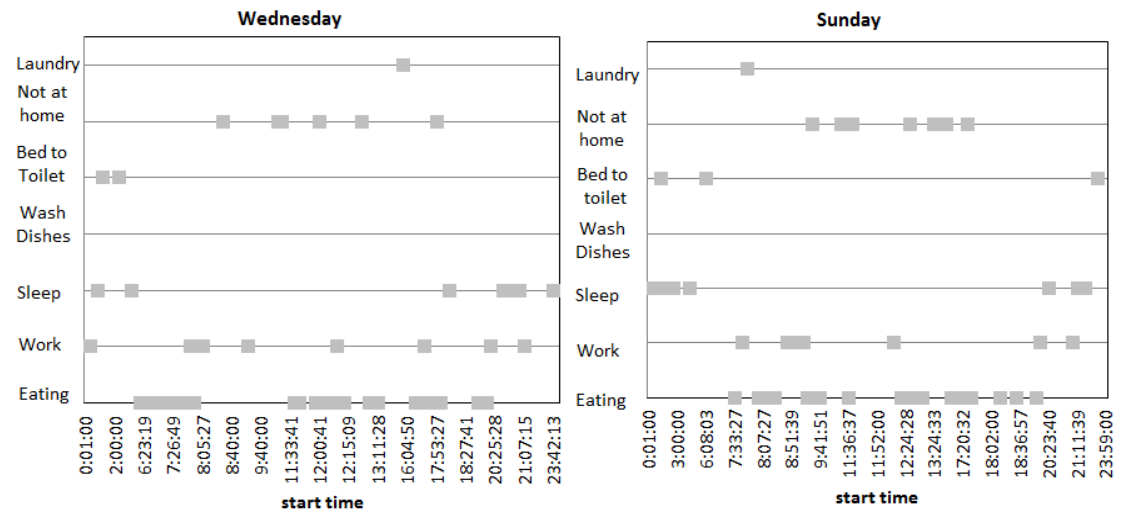

Fig. 2. Start times of the different activities for a period of two months on three different days of the week for subject 2. Different days show different probable activities for similar hours 

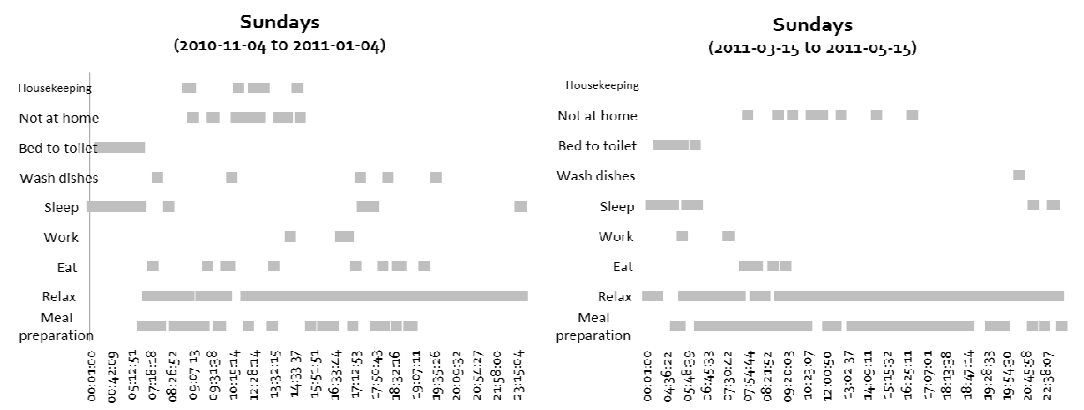

Fig. 3. Start times of the different activities on Wednesdays (top) and Sundays (bottom) for different two-month periods. Routines change from one period (right) to the other

The tests compared the probabilities for weekdays to weekends and for each day against every other day. The z-value for the test is calculated as in eq. (1), where $\hat{p}_{1}$ is the proportion of the activity being tested at day $1, \hat{p}_{2}$ is the proportion of the activity at day 2 and $\mathrm{p}$ is the combined proportion of the activity at both days and is calculated as in eq. (2). $n_{1}$ is the total number of activities on day 1 and $n_{2}$ is the total number of activities of day two for the hour being tested.

$$
\begin{gathered}
z=\frac{\hat{p}_{1}-\hat{p}_{2}}{\sqrt{p(1-p)\left(1 / n_{1}+1 / n_{2}\right)}} \\
p=\frac{x_{1}+x_{2}}{n_{1}+n_{2}}
\end{gathered}
$$

The results of the test show that although most of the days are very similar, for some activities there are significant differences (using p-value $<0.05$ ). For example, when comparing proportions of the week with those of the weekend, the activities at $7 \mathrm{am}$ where found to be different. When comparing Wednesdays and Sundays (as in fig. 2), a difference was found for the activities eating at $6 \mathrm{am}$ and $1 \mathrm{pm}$ (p-value $=0.041)$ and not at home at $8 \mathrm{am}(\mathrm{p}$-value $=0.043)$ and $13 \mathrm{pm}$ and $(\mathrm{p}$-value=0.041). Also, when comparing Mondays with Sundays there are differences at 8 am for eating ( $\mathrm{p}$-value $=0.01)$ and for the activity not at home at $8 \mathrm{pm}(\mathrm{p}$-value $=0.004)$ and at $5 \mathrm{pm}$ ( $\mathrm{p}$-value $=0.02$ ). These experiments allow us to detect temporal patterns and to measure daily and long-term differences in the monitored activities. Nevertheless, a more profound study needs to be done in order to determine if the changes correspond to different routines, alert signs, anomalies or some other factor.

\subsection{Location Patterns}

To study how activities correlate to a location in the house and how this varies for different persons, we explored the frequency each activity happened at the different locations at home. For the first subject, most activities occur mainly at one location, while for the second subject most activities take place at different locations around the house (Fig.4). This means that location could be a good feature for a classifier for the first subject but not for the second subject. 


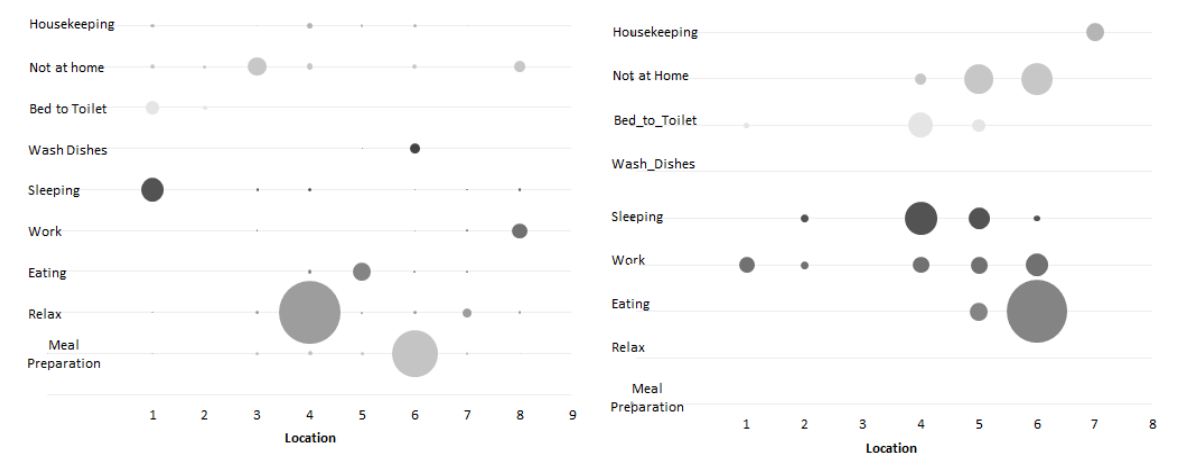

Fig. 4. Frequency (bubble area) of each activity per room in the house (x-axis) for subject 1(left) and subject 2 (right). Not for every person location is a good feature for classification.

\subsection{Frequent Activity Sets}

To analyze frequent activity sequences, we treated the sequence of activities as a string and collected 5-grams ${ }^{3}$. We then found frequent item set using the FP-growth algorithm available at the mahout ${ }^{4}$ library with 5 -grams used as sets. We analyzed patterns for each day of the week for the $2^{\text {nd }}$ subject and for the $1^{\text {st }}$ subject we studied patterns for two periods of two months each separated by 4 months between them.

Visibly, behavior patterns on different days of the week differ even for the same person (Fig. 5) and so do behavior patterns across the year (Fig. 6). In the first case (Fig. 5), the eating activity is not always paired with the not-at-home activity, and it is mostly paired with it on Mondays, Saturdays and Sundays. Also, on Wednesdays and Fridays, eating is not paired with Working, but it is on every other day. Perhaps, the most remarkable example is that eating was only paired with housekeeping on Wednesdays, possibly because that is the day designated for this last activity.

In the second case, most patterns appear in both time periods; however, there is a frequency change from one period to the other (Fig. 6). Some patterns even disappear, for example, the ones that include housekeeping. Classifiers need to adapt to these changes in routines. One challenge that arises from this is identifying when a routine change has occurred instead of an abnormal event.

Finally, to evaluate how pattern changes affect classifiers over time, we trained a hidden Markov Model (HMM) to classify activities of subject. 1 . Then we evaluate its performance with data 1,2,3 and 4 months older than the data the model was trained with. The F-measure for most activities dropped after 4 months compared to the evaluation made with data 1-month after (Fig. 7).

3 For the graphics on this subsection, the following acronyms are used: M: Meal preparation, R: relax, E: eating, W: work, S: sleep, D: Washing Dishes, T: bed to toilet, H: housekeeping N: Not at home.

4 https: //mahout.apache.org/ 


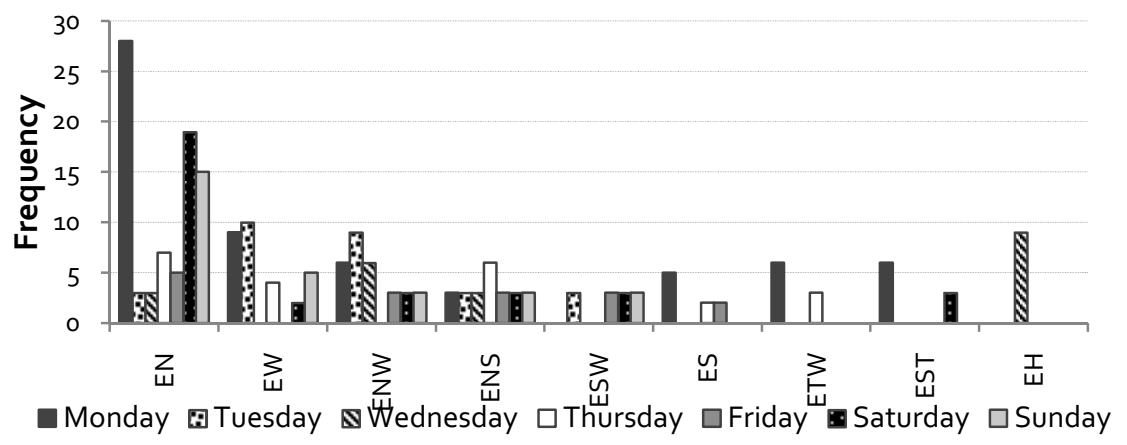

Fig. 5. Frequent activity sets (and frequency per day of the week) for subject 2 . The frequency of each pattern changes according to the day of the week.

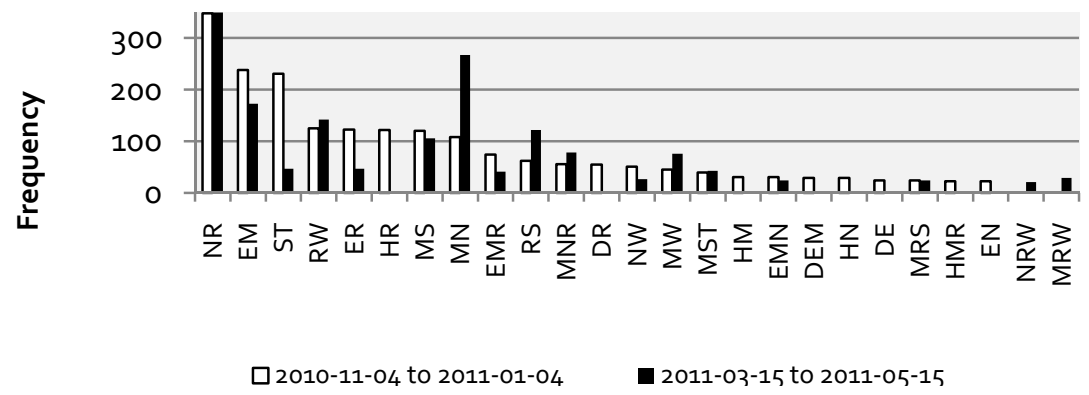

Fig. 6. Frequent activity sets for different two-month periods for the $1^{\text {st }}$ subject. There are differences in the patterns and in the frequencies of the patterns that appear in both periods.
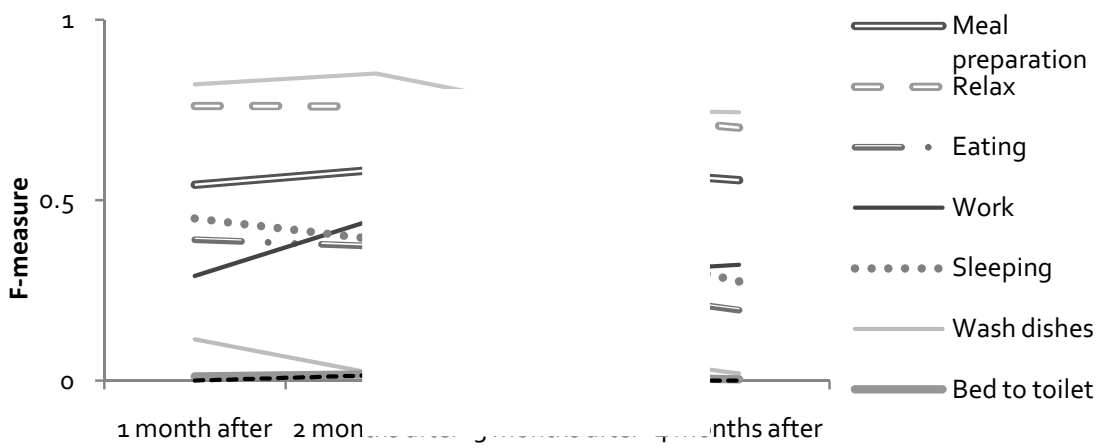

Fig. 7. F-measure for each activity obtained with a HMM trained with 2-month data. For most activities there is a decrease in the f-measure from the first month to the fourth month 


\section{Conclusions and Future Work}

In this paper, we analyzed three behavior patterns: temporal, location and frequent activity sets for two persons in a smart environment. We were able to measure how routines change when analyzed under different contextual variables. We also measured how the fact that patterns change over time, affect the performance of a static model. This implies that static models do not fit well to human activity recognition. Moreover, we evidenced the need for personalized services as different people behave differently. Future work will focus on the use of an algorithm to improve the accuracy of activity recognition. This should help in the creation of better elder care solutions.

Acknowledgement. The authors would like to thank the Google Research Grant that partially financed the presentation of this article.

\section{References}

1. National Institute on Aging: Global Health and Aging (2011)

2. European Commission: Ambient Assisted Living Joint Programme (2008), http: / /www.aal-europe.eu/

3. Lively Inc.: MyLively (2014), http : / /www . mylively. com/

4. Hanson, M.A., Barth, A.T., Silverman, C.: In home assessment and management of health and wellness with BeClose ${ }^{\mathrm{TM}}$ ambient, artificial intelligence. In: Proceedings of the 2nd Conference on Wireless Health, pp. 25:1-25:2. ACM, San Diego (2011)

5. Cook, D.J., Crandall, A.S., Thomas, B.L., Krishnan, N.C.: CASAS: A Smart Home in a Box. IEEE Comput. 46, 62-69 (2013)

6. Munguia Tapia, E.: Activity Recognition in the Home Setting Using Simple and Ubiquitous Sensors (2003)

7. Brush, A.J., Krumm, J., Scott, J.: Activity Recognition Research: The Good, the Bad, and the Future. In: Pervasive 2010 Workshop: How to do Good Research in Activity Recognition, pp. 1-3 (2010)

8. Pruchno, R.A., Rose, M.S.: Time Use by Frail Older People in Different Care Settings. J. Appl. Gerontol. 21, 5-23 (2002)

9. Baltes, M.M., Wahl, H., Schmid-Furstoss, U.: The daily life of elderly Germans: activity patterns, personal control, and functional health. J. Gerontol. 45, 173-179 (1990) 